\title{
Reaction Coupling, Acceptor pK, and Diffusion Control in Light Induced Proton Release of Bacteriorhodopsin
}

\author{
Dietmar Porschke* \\ Max Planck Institut für biophysikalische Chemie, 37077 Göttingen, Germany \\ Received: May 11, 2002; In Final Form: July 27, 2002
}

\begin{abstract}
The mechanism of proton release to the bulk during the photocycle of bacteriorhodopsin has been studied by absorbance measurements using pyranine as indicator. The initial absorbance change of the indicator is characterized by a sigmoidal shape, reflecting coupling of proton pumping with transfer of protons to the indicator. The time constants of pumping $\left(\tau_{1}=20\right.$ to $\left.50 \mu \mathrm{s}\right)$ and of transfer to the indicator $\left(\tau_{2}=0.4\right.$ to 1.5 $\mathrm{ms}$ ) decrease with increasing pyranine concentration, consistent with coupling of an intra- and a bimolecular reaction step. Proton transfer by buffers is dependent on their $\mathrm{pK}$ value. The most efficient buffer in the test proved to be borate; in the presence of $\sim 1 \mathrm{mM}$ borate protons appeared in the bulk with a time constant of $\tau_{2} \approx 60 \mu \mathrm{s}$. The data are described by simple reaction mechanisms. The rate constants of proton transfer are consistent with a standard dependence on the $\mathrm{pK}$ difference between donor and acceptor, but apparently other factors like size and charge of the acceptor contribute as well. The rate constants of proton transfer from bacteriorhodopsin to acceptors with a favorable $\mathrm{pK}$ are at the limit of diffusion control. However, the data indicate the existence of a separate intramolecular reaction step for most acceptors, which seems to reflect proton transfer from the release cavity of bacteriorhodopsin to the periphery for acceptors, which cannot approach this cavity because of steric, electrostatic, and/or other factors. Crystal structures of bacteriorhodopsin support the interpretation that the release site is not directly accessible for acceptors such as pyranine. Proton transfer to borate is without barrier and, thus, there is no general diffusion barrier in the proton release of bacteriorhodopsin.
\end{abstract}

\section{Introduction}

Bacteriorhodopsin is considered to be a "paradigm" of membrane proteins and certainly is among the most thoroughly studied protein molecules. ${ }^{1-4}$ The transformation of light energy into chemical energy in form of a proton concentration gradient is based on a reaction cycle, which has been characterized in considerable detail, mainly by flash spectroscopy. On the basis of spectra in the visible range, six different reaction states $(\mathrm{J}$, $\mathrm{K}, \mathrm{L}, \mathrm{M}, \mathrm{N}$, and $\mathrm{O}$ ) have been identified. Additional substates were characterized by various methods. The release of protons during the $\mathrm{M}$ state of this cycle at the extracellular surface has been studied by $\mathrm{pH}$ indicators. ${ }^{5-14}$ When indicators were fixed covalently on the extracellular surface, protons appeared in times as short as $\sim 70 \mu \mathrm{s}$, whereas indicators in free solution showed proton appearance with time constants of e.g., $1.29 \mathrm{~ms} .^{9,11}$ These results have been used as evidence for the existence of a barrier in the diffusion of protons from bacteriorhodopsin to the bulk solution. Moreover, protons released at the extracellular surface of bacteriorhodopsin disks arrive rapidly at indicators fixed on the intracellular surface, ${ }^{9,11}$ providing evidence for efficient diffusion of protons along the membrane surface. In the general context of energy processing according to Mitchell, ${ }^{15}$ the data seem to support a localized form of a $\mathrm{pH}$ gradient, where protons move directly between a source and a sink, e.g. from a proton pump to $\mathrm{H}^{+}$-ATP synthase, and do not get delocalized by diffusion into the bulk medium.

If there is any barrier against diffusion into bulk solution, what is the nature of this barrier? Nachliel and Gutman ${ }^{16}$ simulated proton transfer from photoactivated bacteriorhodopsin

* Corresponding author. E-mail: dpoersc@gwdg.de. to the bulk by a set differential equations and attribute the delay of protons to a high buffer capacity of the membrane. A completely different conclusion is suggested by early experimental data of Drachev et al., ${ }^{6}$ who report fast appearance of protons in the bulk in the presence of buffers and $p$-nitrophenol as indicator. The issue is of sufficient general interest for further analysis. In the present investigation, proton release in bacteriorhodopsin is analyzed in more detail than previously. The experimental data demonstrate a special form of the release transients that must be expected but has not been detected in previous experiments. These data show coupling of proton pumping by the protein with the subsequent step of proton transfer. Furthermore, the experimental data demonstrate that proton release is very much dependent not only on the concentration but also on the nature of the proton accepting species. The dependence of transfer efficiency on the parameters of the proton acceptor is useful for analysis of a functional structure of the proton release domain in bacteriorhodopsin. Analysis of the data by a simple kinetic model demonstrates that the rate constants of proton transfer follow the expected general dependence on $\mathrm{pK}$ differences. The rate constants for proton transfer observed in cases with a favorable $\mathrm{pK}$ difference are close to the limit of diffusion control and, thus, there is no evidence for the existence of a diffusion barrier. However, the experimental data indicate a separate reaction step, which is suggested to represent proton transfer from an inside cavity of the release site to the periphery of the protein.

\section{Materials and Methods}

Bacteriorhodopsin was prepared as described by Oesterhelt and Stoeckenius ${ }^{17}$ using an overproducing strain (Halobacterium 
salinarum S9) that was kindly provided by Dr. J. Tittor. The sample proved to be homogeneous in SDS gel electrophoresis and in isoelectric focusing. Suspensions of bacteriorhodopsin in given buffers were prepared either by extensive dialysis or by repeated centrifugation into a pellet + resuspension. Most of the present measurements were performed in $50 \mathrm{mM} \mathrm{KCl}$; some measurements were also performed in a low salt medium containing $1 \mathrm{mM} \mathrm{NaClO} 4,0.2 \mathrm{mM} \mathrm{MgSO}_{4}$. The $\mathrm{pH}$ of the samples was adjusted by addition of $\mathrm{NaOH}$ solution from a microsyringe directly into solutions in the measuring cell under nitrogen using an AtmosBag from Aldrich. The cell was then closed under nitrogen. After light adaptation by a Schott cold light source, spectra were measured in this cell with a PerkinElmer Lambda 17. In solutions containing pyranine, $\mathrm{pH}$ values were controlled by recording ratios of absorbances at 454 and $403 \mathrm{~nm}$; contributions to the absorbance from bacteriorhodopsin were subtracted, and the ratio of corrected absorbances at 454 and $403 \mathrm{~nm}$ was used to determine $\mathrm{pH}$ values of solutions in the measuring cell immediately before and after recording data sets of flash spectroscopy. The following reagents were used: sodium azide (Merck, extra pure), ammonium chloride (Merck, $\mathrm{pA})$, boric acid (Baker, pA), cacodylic acid (Sigma), imidazole (Merck), potassium chloride (Baker, pA), 2-( $N$-morpholino)ethanesulfonic acid (MES, Merck)); tris(hydroxymethyl)-aminomethan (TRIS, Merck, pA); pyranine (Molecular Probes).

Flash spectroscopic data were obtained with components from a fluorescence temperature jump instrument. ${ }^{18}$ Solutions were filled into a fluorescence T-jump cell: the central part of this cell was prepared from quartz, the lower electrode was made from $\mathrm{Pt}$, and the Pt electrode closed the cell chamber with an O-ring from perbunan. The optical path lengths in both directions of excitation and absorbance were $7 \mathrm{~mm}$. Flash light was irradiated from a lamp FX1160 (Perkin-Elmer) driven by an EG\&G Electro-Optics model PS302; the wavelength of irradiation was limited to $\lambda>520 \mathrm{~nm}$ by a filter KV 520 from Schott (Mainz, Germany). The flash pulse was recorded by a diode detector. Absorbance changes were measured at $90^{\circ}$ orientation with respect to flash irradiation using a wolfram lamp as light source, a Schoeffel grating monochromator, and a standard photomultiplier detection unit. Detection of scattered flash light by the photomultiplier was avoided by interference filters $(411$ and $458 \mathrm{~nm}$ ) in front of the photomultiplier. The flash light signal and changes in light transmission were recorded by a Tektronix DSA602. Absorbance changes of pyranine, observed at $458 \mathrm{~nm}$, were always corrected for contributions from absorbance changes due to bacteriorhodopsin occurring in the absence of pyranine by subtraction of the signal measured with the same sample at the same $\mathrm{pH}$ in the absence of pyranine. At high concentrations of both pyranine and bacteriorhodopsin, residual signals resulting from the flash pulse passing the 458 $\mathrm{nm}$ interference filter were found due to high amplification of the detector; in these cases the residual flash pulse signals were recorded separately and subtracted. The experimental data were processed (including subtraction of data sets, deconvolution and exponential fitting) by a set of programs designed for analysis of chemical relaxation data. ${ }^{19,20}$ Relaxation time constants were fitted to kinetic models by a combination of a general leastsquares fitting routine based on a simplex procedure ${ }^{21}$ and an algorithm for simulation of relaxation spectra. ${ }^{22}$ Finally, sets of relaxation curves obtained for different concentrations of given acceptors were fitted directly to reaction models ("global" fit) by a combination of a deconvolution procedure, ${ }^{20}$ a routine for calculation of relaxation time constants for given reaction mechanisms ${ }^{22}$ and application of a combination of linear and

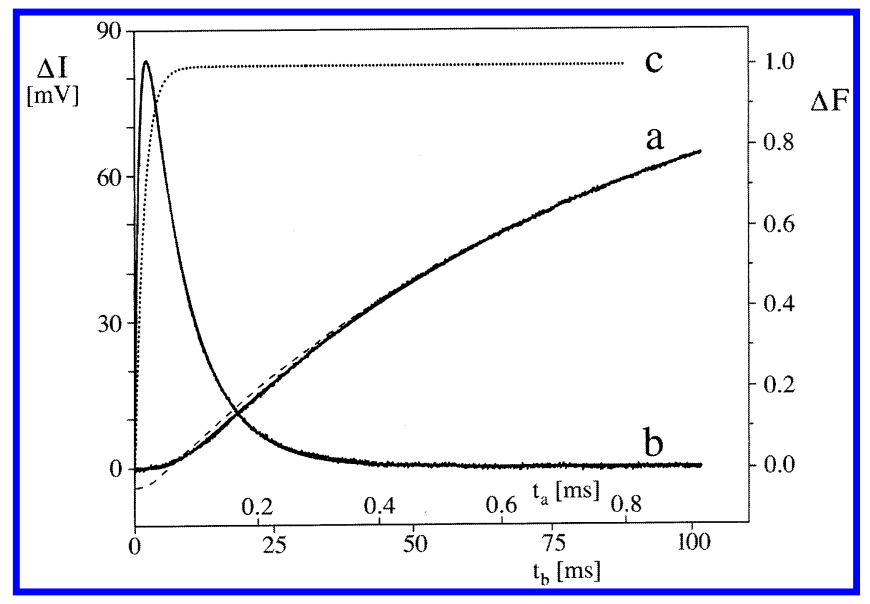

Figure 1. Change of light transmission $\Delta I$ at $458 \mathrm{~nm}$ of a solution containing $19.9 \mu \mathrm{M}$ bacteriorhodopsin and $50.2 \mu \mathrm{M}$ pyranine in 50 $\mathrm{mM} \mathrm{KCl} \mathrm{pH} 8$ induced by irradiation of light pulses $(\lambda>520 \mathrm{~nm})$; traces a and $\mathrm{b}$ (of the same transient) are given at time scales $t_{\mathrm{a}}$ and $t_{\mathrm{b}}$; the transient is averaged from 40 shots and is corrected for contributions from absorbance changes of bacteriorhodopsin measured under identical conditions in the absence of pyranine. Fitting of the transient requires three exponentials: two exponentials for proton release $\left(\tau_{1}=35 \mu \mathrm{s}\right.$; $\left.\tau_{2}=879 \mu \mathrm{s}\right)$ and another one for proton uptake $\left(\tau_{3}=7.64 \mathrm{~ms}\right.$; the line representing the fit cannot be distinguished from the experimental data). The dashed line shows a least-squares fit with two exponentials for comparison. Convolution of the experimental data resulting from the light pulse and from a limited time resolution of the detector was included in the evaluation $(50 \mathrm{mM} \mathrm{KCl}, \mathrm{pH} 7.65)$. The dotted line (c; $\Delta F$ ) shows the reference used for deconvolution ( $\equiv$ intensity of the flash pulse recorded by the detector used for measurements of absorbance changes $\Delta I$, integrated and normalized).

nonlinear fit routines ${ }^{21}$ (for an example of global fitting presented previously cf. ref 23). The reference for deconvolution was obtained by measuring the intensity of the flash light pulse by the detector used for recording pyranine/bacteriorhodopsin transients (at the same bandwidth) and numerical integration.

\section{Results}

Coupling of Proton Pumping and Release to Indicator. The flash light induced change of pyranine absorbance found in the present experiments, indicating proton release at the extracellular side followed by proton uptake at the intracellular side, is of course similar to that published in the literature..$^{5-14}$ However, a careful analysis of the data reveals a feature that has not been described in the literature yet. The initial part of the release signal cannot be represented by a single exponential, because it starts with a zero slope; the shape is sigmoidal, corresponding to a small but noticeable initial delay (Figure 1). This feature is detected in all experimental data, provided that these data are properly corrected for contributions resulting from the photocycle of bacteriorhodopsin recorded in the absence of pyranine. The full time course of proton concentration changes can be described without systematic deviation by a sum of three exponentials, where two exponentials $\left(\tau_{1}\right.$ and $\left.\tau_{2}\right)$ are required to describe proton release.

The explanation of this result is obvious: protons are not released immediately after irradiation: it is well known that about $50 \mu$ s time of the photocycle are required to arrive at the $\mathrm{M}$ state, where protons are released. Calculations of transients based on a two-step reaction scheme, with an intramolecular reaction step representing proton pumping followed by the intermolecular reaction of proton transfer to the indicator (details in section "reaction models"), confirm that the time required for proton pumping gives rise to the sigmoidal appearance of 


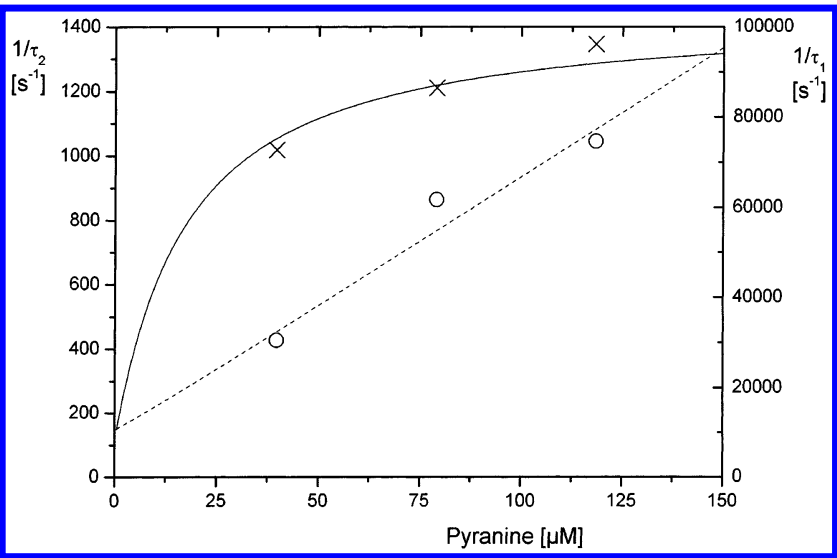

Figure 2. Reciprocal time constants $1 / \tau_{2}\left(\times\right.$, left ordinate) and $1 / \tau_{1}$ $(\mathrm{O}$, right ordinate) as a function of the pyranine concentration at 7.24 $\mu \mathrm{M}$ bacteriorhodopsin. The combined least-squares fit of the $1 / \tau_{1}$ and $1 / \tau_{2}$ values using reaction model $(1)\left(k_{0^{\prime}}^{+}=1.4 \times 10^{3} \mathrm{~s}^{-1}, k_{0^{\prime}}^{-}=9.3 \times\right.$ $\left.10^{3} \mathrm{~s}^{-1}, k_{1^{\prime}}^{+}=6.7 \times 10^{8} \mathrm{M}^{-1} \mathrm{~s}^{-1}\right)$ is represented by a dashed and a continuous line for $\tau_{1}$ and $\tau_{2}$, respectively.

the proton release curves. Thus, the sigmoidal shape of the release curves must be expected and clearly is not an artifact resulting from insufficient instrumentation.

Measurements under exactly the same experimental conditions recorded at $411 \mathrm{~nm}$, where the rise of the $M$ state is observed, do not show the delay found in the pyranine transients at $458 \mathrm{~nm}$. This result is in agreement with literature data and demonstrates that the delay found at $458 \mathrm{~nm}$ is not due to any problem with instrumentation and/or deconvolution.

Measurements at different concentrations of pyranine clearly demonstrate an acceleration of the relaxation with increasing concentrations. This concentration dependence can be measured more easily for the slower of the release time constants $\tau_{2}$ for technical reasons, but the experimental data also indicate a concentration dependence of $\tau_{1}$ (Figure 2). A serious problem for determination of accurate data is the strong dependence of the transients on $\mathrm{pH}$. Although the sample cell was closed under nitrogen (cf. Methods), there used to be a slow $\mathrm{pH}$ drift, apparently due to diffusion of $\mathrm{CO}_{2}$ into the cell. Because of the low reactant concentrations the buffering capacity was very low and, thus, small quantities of $\mathrm{CO}_{2}$ were sufficient to cause a $\mathrm{pH}$ drift. It is difficult to estimate the error level remaining from the problem of $\mathrm{pH}$ control. However, the errors in the final rate constants (cf. Table 1) estimated from reproducibility are probably mainly due to residual shifts of $\mathrm{pH}$ values.

Dependence of Proton Release on Proton Acceptors. The rate of proton transfer may depend on various factors, including the structure of the proton acceptor. To check for any dependence of this type, the appearance of protons in solution has
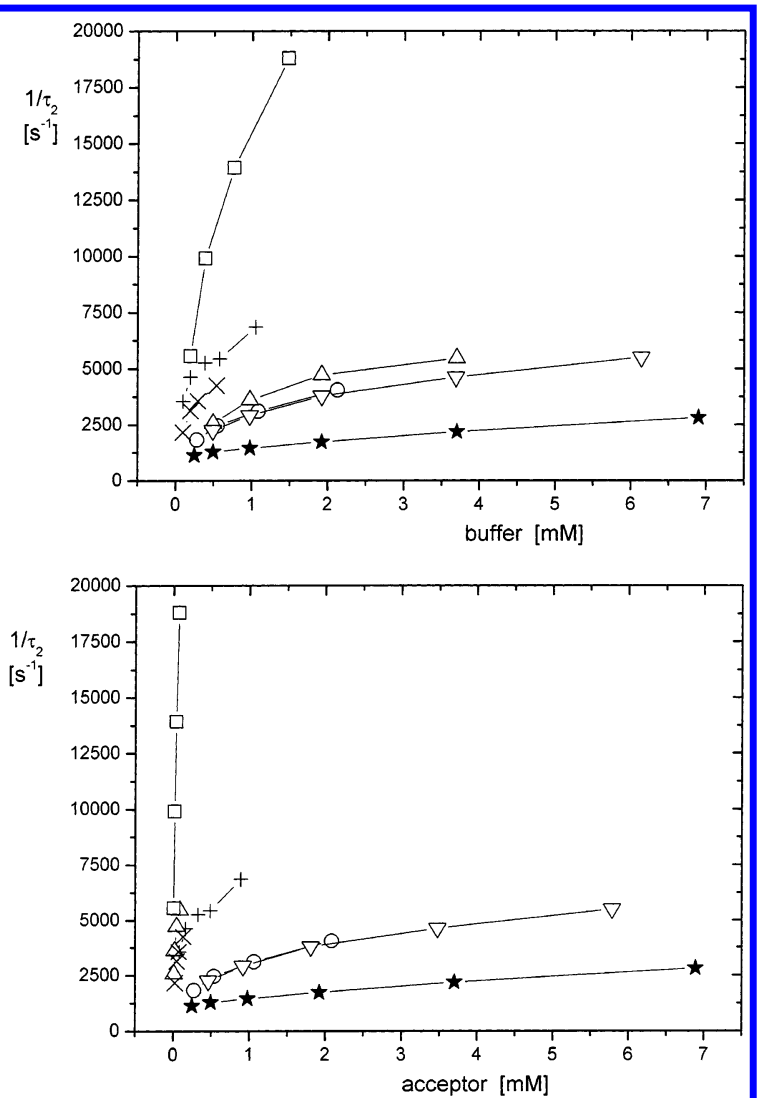

Figure 3. Reciprocal time constants $1 / \tau_{2}$ as a function of the concentration of different buffers: azide $(\star)$, cacodylate $(\nabla)$, MES $(\bigcirc)$, imidazole $(+)$, TRIS $(\times)$, borate $(\square)$ and ammonium $(\triangle)(50 \mathrm{mM} \mathrm{KCl}$; data for cacodylate in $1 \mathrm{mM} \mathrm{NaClO}_{4}, 0.2 \mathrm{mM} \mathrm{MgSO}_{4}$ ). (a) Abscissa with the total buffer concentration. (b) Abscissa with the concentration of the proton acceptor species of the respective buffer at the given $\mathrm{pH}$.

been measured in the presence of different acceptor species. In all cases pyranine was used as indicator, because its spectral parameters are very convenient for analysis of proton release from bacteriorhodopsin.

Added proton acceptors serve as catalysts for transfer of protons to the indicator. As shown in Figure 3, clear differences in the transfer activity are found for various buffer species. A particularly high transfer efficiency is observed for borate.

Because protons are accepted from bacteriorhodopsin only by the deprotonated buffer species, the data of Figure 3a, which are presented with the total buffer concentration in the abscissa, have been plotted again in Figure $3 b$ as a function of the concentration of the unprotonated buffer species at the given $\mathrm{pH}$ value. In this plot the position of borate is more extreme,

TABLE 1: Rate Constants $k_{0}^{+}, k_{0}^{-}, k_{1}^{+}$, and $k_{3}^{+}$Obtained According to the Cyclic Reaction Scheme 2 by Global Fitting ${ }^{a}$

\begin{tabular}{llccccccc}
\hline acceptor & $\mathrm{pK}$ & $\mathrm{pH}$ & $\begin{array}{c}\text { salt } \\
{[\mathrm{mM}]}\end{array}$ & $\begin{array}{c}k_{0}^{+} \\
{\left[\mathrm{s}^{-1}\right]}\end{array}$ & $\begin{array}{c}k_{0}^{-} \\
{\left[\mathrm{s}^{-1}\right]}\end{array}$ & $\begin{array}{c}k_{1}^{+} \\
{\left[\mathrm{M}^{-1} \mathrm{~s}^{-1}\right]}\end{array}$ & $\begin{array}{c}k_{2}^{+} \\
{\left[\mathrm{M}^{-1} \mathrm{~s}^{-1}\right]}\end{array}$ \\
\hline azide & 4.7 & 7.66 & 1 & $4.1 \times 10^{3}$ & $2.0 \times 10^{4}$ & $3.9 \times 10^{6}$ & $1.8 \times 10^{8}$ \\
azide & 4.7 & 7.96 & 50 & $4.6 \times 10^{3}$ & $2.0 \times 10^{4}$ & $7.3 \times 10^{6}$ & $2.5 \times 10^{8}$ & $1.0 \times 10^{9}$ \\
MES & 6.1 & 7.45 & 50 & $6.1 \times 10^{3}$ & $2.0 \times 10^{4}$ & $1.8 \times 10^{7}$ & $1.5 \times 10^{8}$ & $1.0 \times 10^{9}$ \\
cacodylate & 6.3 & 7.51 & 1 & $5.4 \times 10^{3}$ & $2.0 \times 10^{4}$ & $1.7 \times 10^{7}$ & $2.1 \times 10^{8}$ & $1.0 \times 10^{9}$ \\
imidazole & 7 & 7.73 & 50 & $7.3 \times 10^{3}$ & $2.0 \times 10^{4}$ & $1.7 \times 10^{8}$ & $1.2 \times 10^{8}$ & $1.0 \times 10^{9}$ \\
TRIS & 8.21 & 7.73 & 50 & $6.4 \times 10^{3}$ & $2.0 \times 10^{4}$ & $2.5 \times 10^{8}$ & $1.3 \times 10^{8}$ & $4.0 \times 10^{8}$ \\
borate & 9.14 & 7.98 & 50 & $1.4 \times 10^{4}$ & $3.0 \times 10^{3}$ & $4.9 \times 10^{8}$ & $4.4 \times 10^{7}$ & $1.0 \times 10^{8}$ \\
ammonia & 9.4 & 7.87 & 50 & $7.8 \times 10^{3}$ & $2.0 \times 10^{4}$ & $7.1 \times 10^{8}$ & $1.0 \times 10^{8}$ & $5.0 \times 10^{7}$ \\
pyranine & 7.3 & 8.04 & 50 & $2.4 \times 10^{3}$ & $2.0 \times 10^{4}$ & $1.1 \times 10^{9}$ & $2.2 \times 10^{8}$ & $1.0 \times 10^{9}$
\end{tabular}

${ }^{a}$ The rate constants $k_{2}^{+}$were estimated from the known dependence of proton-transfer rate constants on the $\mathrm{p} K$-difference. Estimated accuracy $k_{0}^{+} \pm 10 \% ; k_{0}^{-}, k_{1}^{+}$and $k_{3}^{+} \pm 30 \%$. 

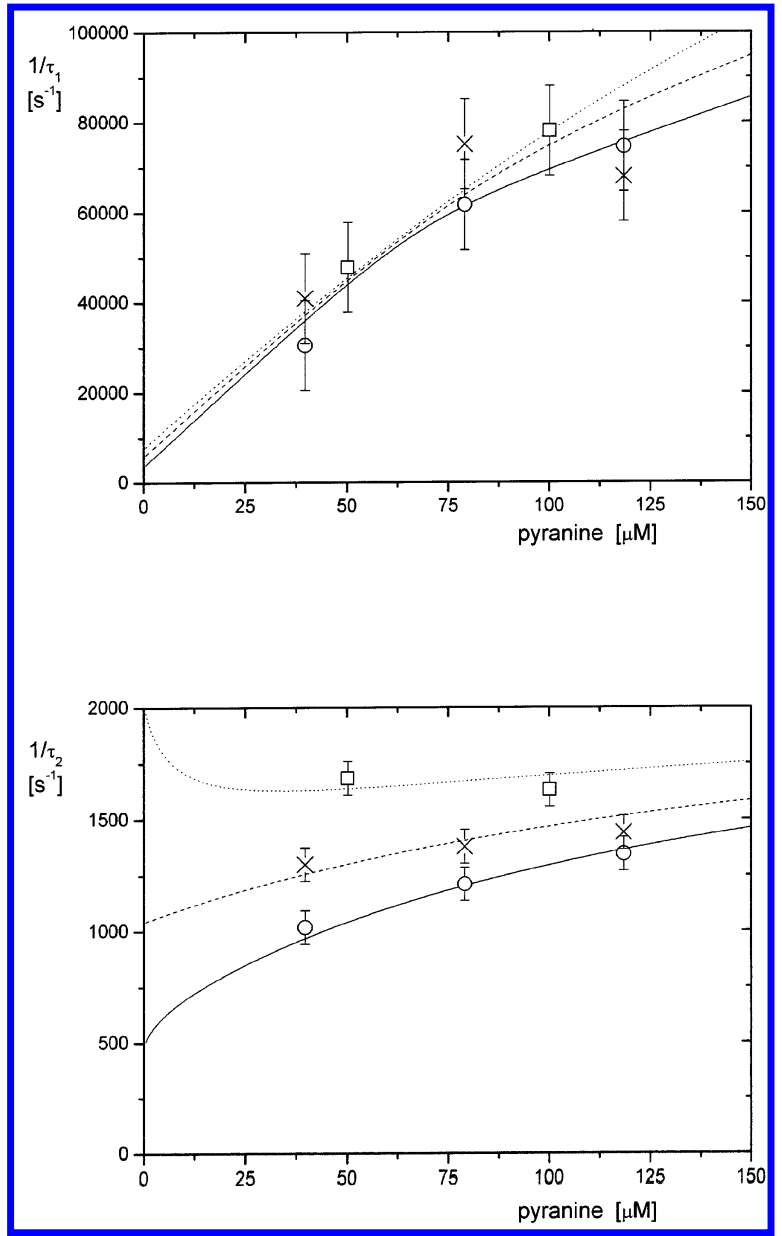

Figure 4. Reciprocal time constants $1 / \tau_{1}$ (a) and $1 / \tau_{2}$ (b) as a function of the pyranine concentration at three different bacteriorhodopsin concentrations (7.24 $\mu \mathrm{M} \bigcirc ; 15.7 \mu \mathrm{M} \times ; 30.2 \mu \mathrm{M} \square)$ in $50 \mathrm{mM} \mathrm{KCl}$ at $\mathrm{pH}$ 8.03. The combined least-squares fit of all $1 / \tau_{1}$ and $1 / \tau_{2}$ values according to reaction model $3\left(k_{0}^{+}=2.2 \times 10^{3} \mathrm{~s}^{-1} ; k_{0}^{-}=3 \times 10^{4} \mathrm{~s}^{-1}\right.$; $k_{1}^{+}=1.3 \times 10^{9} \mathrm{M}^{-1} \mathrm{~s}^{-1} ; k_{3}^{+}=3.3 \times 10^{8} \mathrm{M}^{-1} \mathrm{~s}^{-1}$ ) is represented by continuous, dashed, and dotted lines for bacteriorhodopsin concentrations of $7.24,15.7$, and $30.2 \mu \mathrm{M}$, respectively.

closely followed by ammonia. As should be expected, the $\mathrm{pK}$ value of the proton acceptor is essential.

Dependence of Proton Transfer on Bacteriorhodopsin Concentration. The experimental data demonstrate a clear decrease of $\tau_{2}$ with increasing bacteriorhodopsin concentration (Figure 4), even though pyranine was always in excess of bacteriorhodopsin, and thus a dependence on the concentration of bacteriorhodopsin is not expected to be detectable at the given limits of experimental accuracy. Simulations based on a simple two step-reaction scheme described below (1) confirm this expectation.

Thus, it may be suspected that the experimental dependence of $\tau_{2}$ on the bacteriorhodopsin concentration is due to a contamination by some proton transferring species. Standard procedures used for purification of bacteriorhodopsin may not eliminate such contamination at the micromolar concentration level. However, bacteriorhodopsin samples have been dialyzed extensively and/or resuspended in solutions that had been freshly degassed in order to avoid proton transfer via any buffer of low molecular weight, including hydrogencarbonate ions. These purification steps did not affect the relaxation effects associated with proton release. Thus, it is possible that the dependence on the bacteriorhodopsin concentration is due to a special proton- transfer pathway via acceptor/donor groups on bacteriorhodopsin disks.

Reaction Models. A reaction scheme must include at least two reaction steps: (1) proton pumping within bacteriorhodopsin, which can be represented for the present purpose by a single reaction step; and (2) proton transfer from bacteriorhodopsin to pyranine

$$
\begin{gathered}
\mathrm{BR} \underset{k_{0^{\prime}}}{\stackrel{k_{0^{\prime}}^{+}}{\rightleftharpoons}} \mathrm{BH} \\
\mathrm{BH}+\mathrm{I} \underset{k_{1^{\prime}}}{\stackrel{k_{1^{\prime}}^{+}}{\rightleftharpoons}} \mathrm{B}+\mathrm{IH}
\end{gathered}
$$

where $\mathrm{BR}, \mathrm{BH}$, and $\mathrm{B}$ represent the resting state of bacteriorhodopsin, the states with a proton and without a proton at the release site, respectively; I and $\mathrm{IH}$ represent the unprotonated and the protonated states of the indicator, respectively. This model is expected to be sufficient in the absence of added buffers. Some parameters of the reaction are known: the $\mathrm{p} K$ of the indicator pyranine ${ }^{24}$ is 7.3 and the $\mathrm{p} K$ of the proton release group of bacteriorhodopsin has been reported ${ }^{13}$ to be 5.8 . These pK values define the equilibrium constant $k_{1^{\prime}}^{+} / k_{1^{\prime}}^{-}$of the second reaction step. Fitting of the two time constants resulting from the reaction scheme to the set of experimental data shown in Figure 2 provided the remaining three parameters. The rate constant of proton transfer $k_{1^{\prime}}^{+}=6.7 \times 10^{8} \mathrm{M}^{-1} \mathrm{~s}^{-1}$ is in the expected range, whereas the ratio of the rate constants $k_{0^{\prime}}^{+}=$ $1.4 \times 10^{3} \mathrm{~s}^{-1}$ and $k_{0^{\prime}}^{-}=9.3 \times 10^{3} \mathrm{~s}^{-1}$ is unexpectedly low for an "irreversible" reaction. This problem will be discussed below. Based on the reproducibility of the data, the accuracy of these rate constants is estimated to be $\pm 10 \%$ for $k_{1^{\prime}}^{+}$and $\pm 20 \%$ for $k_{0^{\prime}}^{+}$and $k_{0^{\prime}}^{-}$.

A quantitative analysis of the relaxation curves measured in the presence of buffers must be based on a reaction scheme including the major reactions contributing to the proton transfer. As discussed by Eigen, ${ }^{25}$ reactions based on "free" protons or on defect protons $\left(\mathrm{HO}_{\mathrm{aq}}^{-}\right)$do not provide any essential contribution in the $\mathrm{pH}$ range around 7 under usual conditions because of their low concentrations. The remaining transfer reactions expected in solutions containing bacteriorhodopsin, indicator $\mathrm{I}$, and transfer reagent $\mathrm{T}$ are included in the following cyclic reaction scheme:

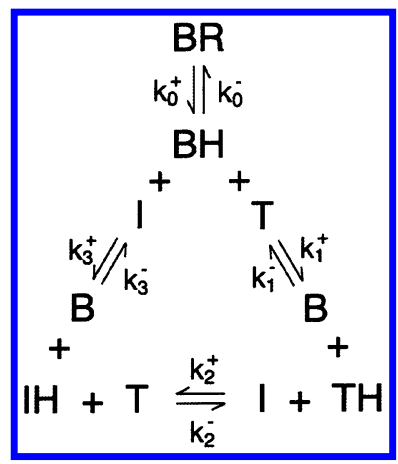

The initial step $\mathrm{BR} \rightleftharpoons \mathrm{BH}$ represents light induced proton pumping (rate constants $k_{0}^{+}$and $k_{0}^{-}$) and is coupled to three different proton-transfer reactions: (1) the pumped proton may be transferred from bacteriorhodopsin $(\mathrm{BH})$ to the unprotonated transfer reagent $\mathrm{T}$ (rate constants $k_{1}^{+}$and $k_{1}^{-}$); (2) the protonated transfer reagent TH may pass its proton to the indicator I (rate constants $k_{2}^{+}$and $k_{2}^{-}$); (3) the pumped proton may also 
be transferred from the state $\mathrm{BH}$ to the indicator I directly (rate constants $k_{3}^{+}$and $k_{3}^{-}$).

The number of parameters for this reaction scheme is rather high and, thus, it is difficult to determine exact values for all the rate constants from the limited set of the available experimental data. However, analysis is favored by the fact that some independent information is available. First of all, the pK values are known and, thus, equilibrium constants can be calculated. Furthermore, the transfer rate between buffer and indicator may be estimated from the known general dependence of rate constants on $\mathrm{pK}$ differences. ${ }^{25}$ The rate constant $1 \times$ $10^{9} \mathrm{M}^{-1} \mathrm{~s}^{-1}$ observed for proton transfer from acetic acid to imidazole is used as a reference for the diffusion controlled limit. ${ }^{25}$ Thus, the number of unknown parameters is reduced considerably. Further reduction would be possible if the parameters obtained from the data for bacteriorhodopsin + pyranine in the absence of buffer could be introduced into the analysis of the data measured in the presence of buffer. However, as discussed below, fitting according to model (2) revealed coupling between the parameters of the bimolecular proton transfer and the parameters of the initial intramolecular reaction step; thus, simple substitution appeared to be impossible. Because of this coupling, each set of experimental data was fitted independently and parameters were not fixed with two exceptions: pK values and the transfer rate between buffer and indicator.

The data obtained for different buffers show individual fitting qualities. In the case of borate the data do not provide information on a specific value for $k_{0}^{-}$. The experimental time constants are compatible with a range of $k_{0}^{-}$values as long as $k_{0}^{-} \ll k_{0}^{+}$. This result is expected for an irreversible reaction. Photoinduced pumping of protons in bacteriorhodopsin is irreversible, because the proton does not return to its original donor.

The data obtained for other buffers provide evidence for a different and more complex reaction mechanism. Fitting of these data indicates $k_{0}^{-} \geq k_{0}^{+}$, corresponding to an equilibrium reaction between $\mathrm{BR}$ and $\mathrm{BH}$, with a higher population of the initial state BR. This is in contrast with expectation because the photoinduced net output of protons suggests the absence of any detectable back-reaction.

Global Fitting. The usual procedure for fitting of relaxation data involves two steps. First, relaxation time constants are fitted to relaxation curves and in a separate second step these time constants are fitted to reaction models. This procedure is useful for assignment of the minimal mechanism required to describe the experimental data. However, for a final evaluation of parameters it is more useful to fit the rate constants of the reaction model directly to the set of relaxation curves measured at different reactant concentrations. This "global fit" procedure avoids problems with assignment of proper statistical weights for different relaxation processes. In the present case $\tau_{1}$ values cannot be assigned as accurately as $\tau_{2}$ values, because $\tau_{1}$ is derived from a relatively small part of the relaxation curves. Appropriate statistical weights are assigned automatically during global fitting. An example of a global fit is shown in Figure 5. In almost all cases the experimental data could be fitted by the cyclic reaction mechanism at a satisfactory accuracy (exception: azide; cf. below), and in almost all cases the results obtained by global fitting are consistent with those obtained by the two step fitting procedure. Some change in the resulting parameters was observed in the case of TRIS buffer. Because global fitting is expected to be more reliable, the discussion presented below is based on parameters from global fits.

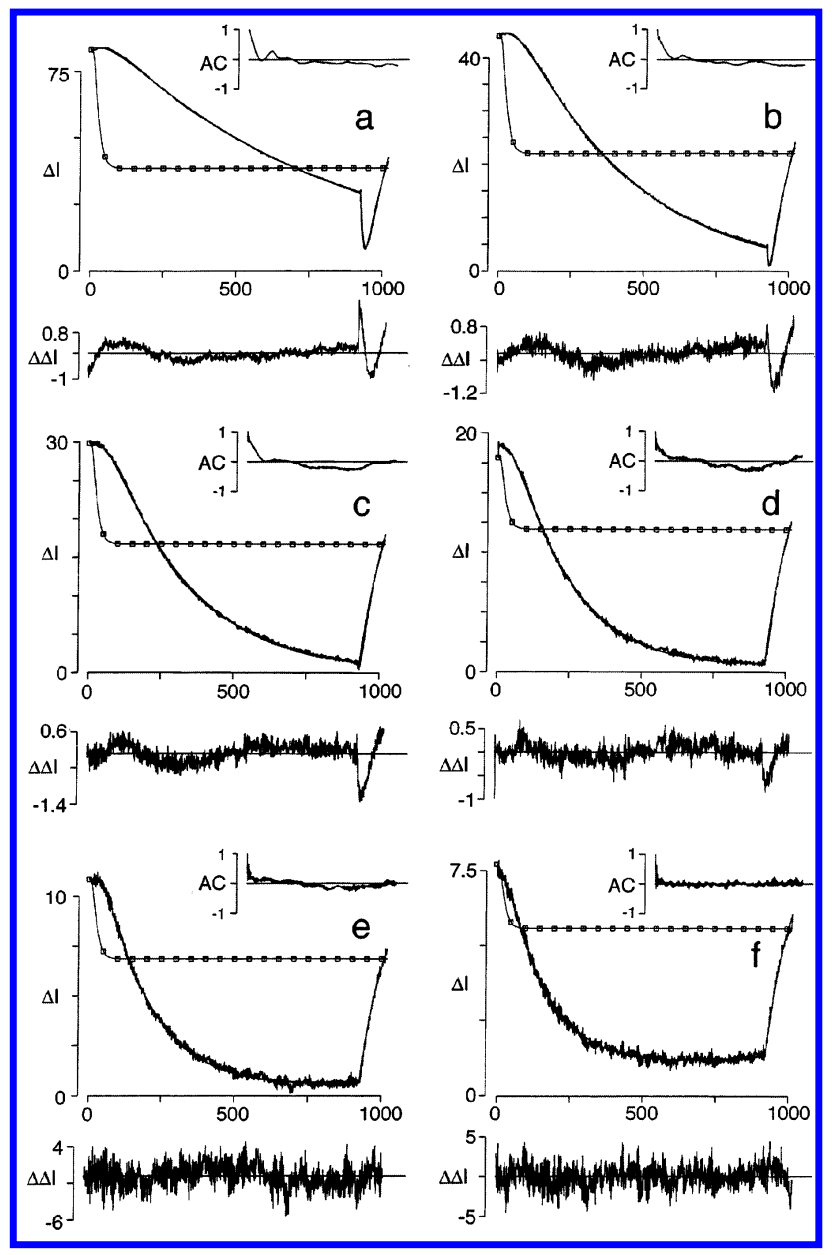

Figure 5. Global fit of proton release curves indicated by absorbance changes of pyranine at $458 \mathrm{~nm}$ in the presence of different concentrations of $\mathrm{NH}_{4} \mathrm{Cl}$. In each panel the line with noise represents the measured change of light intensity $\Delta I$ in $\mathrm{mV}$; a line without noise representing the least-squares fit cannot be distinguished from the experimental data. The residuals $\Delta \Delta I$ are given separately below and the autocorrelations $\mathrm{AC}$ of the residuals are given as insets. The lines marked by open squares represent the reference used for deconvolution. The ordinates $\Delta I$ and $\Delta \Delta I$ are in $\mathrm{mV}$; the abscissas are given in "channels"; the time interval between individual channels is $1 \mu$ s up to channel 924 and $100 \mu$ s for channels 924 to 1020 . Other conditions: $20{ }^{\circ} \mathrm{C} ; 50 \mathrm{mM} \mathrm{KCl} ; 13.3 \mu \mathrm{M}$ bacteriorhodopsin, $50.2 \mu \mathrm{M}$ pyranine, $\mathrm{pH}$ 7.87. Concentrations of $\mathrm{NH}_{4} \mathrm{Cl}$ in $\mathrm{mM}$ are (a) 0 ; (b) 0.248 ; (c) 0.539 ; (d) 1.111 ; (e) 2.216 ; (f) 4.28 . The last relaxation process representing proton uptake (cf. Figure 1) was not described by the reaction model (2) but by fixed time constants obtained from fitting of single relaxation curves. Because the final part of the relaxation curves $(t>10.5 \mathrm{~ms})$ does not contribute to the information on proton release, it was not included in the global fit. The complete set of data used for global fitting is shown in this figure, corresponding to a total recording time of $10.5 \mathrm{~ms}$ after light pulse initiation. The fitted parameters are given in Table 1 . The relatively large $\Delta \Delta I$ values in panels a to $\mathrm{c}$ at channels $>924$ are due to jitter in the time base caused by the large change in the time interval.

The differences in the concentration dependences of the relaxation curves obtained for different buffers are clearly reflected in the parameters specified by minima in the error sum. In cases such as $\mathrm{NH}_{4}$, borate, and TRIS, a minimum in the error sum is found for each of the rate constants to be evaluated and, thus, all parameters can be determined. In other cases the information is limited due to coupling of the rate constants $k_{0}^{-}$ and $k_{1}^{+}$: an increase in the error sum due to a change of $k_{0}^{-}$can be compensated by a change of $k_{1}^{+}$. Thus, these data sets are consistent with a relatively broad range of $k_{0}^{-}$values. Because 


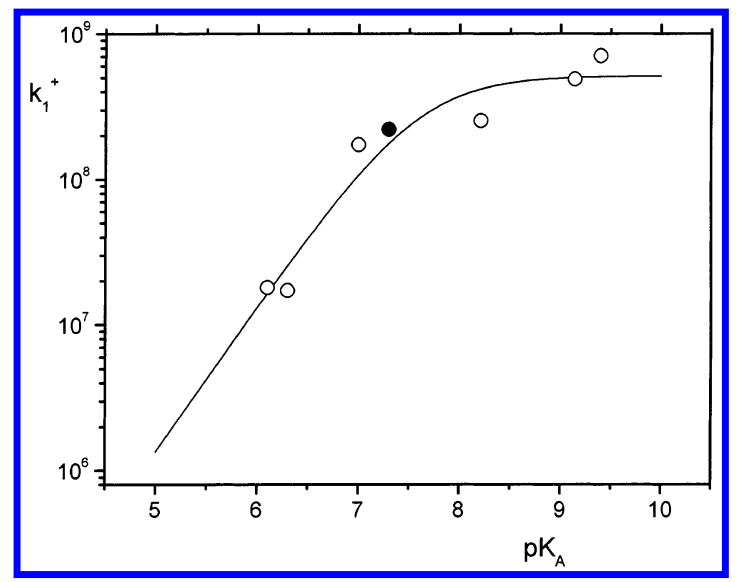

Figure 6. $k_{1}^{+}$obtained for different buffers as a function of the $\mathrm{pK}$ value of the acceptor. The data indicated by open circles $(O)$ were used for a least-squares fit to eq 3 ; the continuous line shows the resulting fit with $k_{1, \max }^{+}=5.2 \times 10^{8} \mathrm{M}^{-1} \mathrm{~s}^{-1}$ and $\mathrm{pK}_{\mathrm{D}}=7.58$. The $k_{1}^{+}$value obtained for pyranine (๑) was not included in the fit (note that in this case $k_{3}^{+}$of reaction scheme 2 reflects direct proton transfer to pyranine).

some rate constants of the cyclic reaction scheme, including $k_{0}^{-}$, are expected to be constant in the presence of different buffers, the corresponding rate constants should be identical. Thus, the data obtained for different buffers were examined for satisfactory fits by a set of parameters with identical values for identical reactions. For most of the buffers, fitting with such a set of parameters was possible. However, parameters deviating from the consensus set were found in the case of borate.

The rate constants obtained by fitting of experimental data to reaction scheme (2), compiled in Table 1, demonstrate a clear dependence of the proton-transfer rate $k_{1}^{+}$to the acceptor on its $\mathrm{pK}$ value. As should be expected, high $k_{1}^{+}$values are observed when the acceptor $\mathrm{pK}$ is high, and thus proton transfer can be at the diffusion controlled limit. The $k_{1}^{+}$values decrease with decreasing $\mathrm{pK}$ value of the acceptor, when the proton has to be transferred against an increasing free energy barrier. These data are consistent with the general rules for proton-transfer reactions described by Eigen. ${ }^{25}$ The transition between the diffusion controlled regime $\left(\mathrm{pK}_{\mathrm{D}}-\mathrm{pK}_{\mathrm{A}}<1\right)$, and the regime controlled by the energy barrier $\left(\mathrm{pK}_{\mathrm{D}}-\mathrm{pK}_{\mathrm{A}}>1\right)$ is described by the following equation on the basis of the free energy differences

$$
k_{1}^{+}=\frac{k_{1, \max }^{+}}{1+10^{\mathrm{pK}_{\mathrm{D}}-\mathrm{pK}}}
$$

where $\mathrm{pK}_{\mathrm{D}}$ and $\mathrm{pK}_{\mathrm{A}}$ are the $\mathrm{pK}$ values of the bacteriorhodopsin donor site and of the acceptor, respectively, and $k_{1, \max }^{+}$is the maximal rate constant in the limit of a favorable $\mathrm{pK}$ difference. When this equation is used to fit experimental rate constants of proton transfer between acetic acid as donor and different acceptors (hydrazine, imidazole, aniline, formiate and $\mathrm{m}$ chloroaniline ${ }^{25,26}$ ) the resulting $\mathrm{pK}$ value of the donor is 4.9 (agreement with the literature value 4.8 for acetic acid within the limits of accuracy).

As shown in Figure 6 , the $k_{1}^{+}$values for proton transfer between bacteriorhodopsin and a wide variety of buffers can be described by the expected dependence on the $\mathrm{pK}$ difference. The value $\mathrm{pK}_{\mathrm{D}}=7.6$ of the donor group obtained from the least-squares fit using eq 3 is clearly larger than that given previously $^{13}$ for the release group $(\mathrm{pK}=5.8)$. Apparently the difference is due to the fact that the $\mathrm{pK}$ determined in the present investigation represents a kinetic pK value (cf. Discussion).
The $k_{1}^{+}$value obtained for azide on the basis of reaction scheme (2) is about an order of magnitude larger than expected from the dependence shown in Figure 6. This deviation is hardly due to experimental uncertainty but provides evidence for the existence of a separate proton-transfer pathway, which contributes in the presence of azide because of its unfavorable $\mathrm{pK}$ value. The existence of an additional reaction pathway is also indicated by systematic deviations in the global fit of the relaxation curves measured in the presence of azide. Special effects associated with azide ions have been observed in previous investigations. ${ }^{27}$

BR Concentration Dependence. The data for the proton transfer between bacteriorhodopsin and pyranine obtained at low concentrations of bacteriorhodopsin $c_{\mathrm{BR}}$ can be described by a two-step reaction mechanism (cf. Figure 2). However, as discussed above, an additional reaction pathway must be included at high $c_{\mathrm{BR}}$ values. The complete set of experimental data may be described quantitatively on the basis of reaction scheme (2), assuming that the transfer reagent $\mathrm{T}$ is equivalent to some residues at the surface of bacteriorhodopsin and using a concentration for the species $\mathrm{T}$ corresponding to the concentration of bacteriorhodopsin. The $\mathrm{pK}$ value of the surface residue was assumed to be 6 . As shown in Figure 4, the concentration dependence of both $\tau_{1}$ and $\tau_{2}$ can be represented by this mechanism with high accuracy. The rate constants obtained from this fit for the pumping reaction and for the proton transfer to the indicator are in the same range as those obtained from the data measured at low concentrations, but some change of the values indicates that the additional reaction included in scheme (3) contributes to some degree already at the lower concentration used in Figure 2. The results obtained by the standard fitting procedure are confirmed by global fitting.

Extension of the Reaction Mechanism. As discussed above, the results obtained by the reaction schemes (1) and (2) suggest that the reaction mechanism is more complex. A simple extension of the mechanism (1) may be used as an example

$$
\begin{gathered}
\mathrm{BR} \underset{k_{0^{*}}^{-}}{\stackrel{k_{0^{*}}^{+}}{\rightleftharpoons}} \mathrm{BA} \underset{k_{1^{*}}^{-}}{\stackrel{k_{1^{*}}^{+}}{\rightleftharpoons}} \mathrm{BH} \\
\mathrm{BHR}+\mathrm{T} \underset{k_{2^{*}}^{\stackrel{k_{2^{*}}^{+}}{+}}}{\mathrm{B}}+\mathrm{TH}
\end{gathered}
$$

where the resting state BR of bacteriorhodopsin is first converted to an activated state BA and subsequently BA is converted to the state $\mathrm{BH}$ with the proton exposed for transfer to the acceptor T. For a least-squares fit of the experimental data shown in Figure 2, the rate constants of the first reaction were fixed to $k_{0^{*}}^{+}=2 \times 10^{4} \mathrm{~s}^{-1}$ and $k_{0^{*}}^{-}=10 \mathrm{~s}^{-1}$, corresponding to a relaxation time constant of $50 \mu \mathrm{s}$. The resulting rate constants $k_{1^{*}}^{+}=1.4 \times 10^{3} \mathrm{~s}^{-1}, k_{1^{*}}^{-}=9.6 \times 10^{3} \mathrm{~s}^{-1}$, and $k_{2^{*}}^{+}=6.8 \times 10^{8}$ $\mathrm{M}^{1} \mathrm{~s}^{-1}$ are similar to those obtained for the corresponding reaction steps of scheme (1). The quality of the fit is also very similar. Thus, the intramolecular reaction step of reaction scheme (1) may be separated into two steps, where the first one in scheme (4) is virtually irreversible and the parameters of the second step in scheme (4) are almost identical with those of the first step derived on the basis of scheme (1).

\section{Discussion}

Coupling of Reactions in Proton Release. Because the mobility of protons in water is known to be particularly high, the interpretation of experimental data initially was based on the assumption that protons are transferred directly via diffusion through the aqueous phase. ${ }^{9,11}$ However, the concentration of 
free protons in aqueous solution is minimal at $\mathrm{pH}$ values around 7 and, thus, protons are usually transferred in aqueous solutions either directly from donors to acceptors or via buffers serving both as proton acceptors and donors at a high efficiency. Direct proton transfer between bacteriorhodopsin and indicator was included in a model presented by Nachliel and Gutman, ${ }^{16}$ but some of the rate constants used in their model are not consistent with experimental data. General rules for the kinetics of proton transfer were discussed by Eigen. ${ }^{25}$ Analysis and description of proton release in the present investigation is consistent with these rules.

Any quantitative analysis of proton release from bacteriorhodopsin to the bulk requires that coupling between the first intramolecular step of pumping and the subsequent bimolecular step of proton transfer into the bulk is described explicitly. Pumping is not infinitely fast but is known to take about $50 \mu \mathrm{s}$ and, thus, the pumping time affects the appearance of protons in solution. Coupling between the two reactions is reflected by a special sigmoidal form of the release curves, which has neither been described nor discussed in previous investigations.

Numerical simulations based on the coupled reaction scheme (1) demonstrate that the delay does not appear for large perturbations, i.e., large changes of the $\mathrm{pH}$ induced by extensive proton production exceeding the buffering capacity of the system. In this case the observed time constants cannot be interpreted by usual models anymore, because the reaction time constants are strongly $\mathrm{pH}$ dependent. Another potential reason for missing the delay effect is incorrect compensation of the absorbance changes resulting from bacteriorhodopsin at the wavelength used for recording the indicator signal.

The present experimental data were obtained by a technique without expensive lasers. A disadvantage of this technique is a limited time resolution. However, a direct comparison of experimental transients in the 10 to $50 \mu$ s time range shows that the signal-to-noise ratio of the measurements in the present investigation is at least an order of magnitude higher than that in previous investigations. Thus, it is very likely that the delay effect was simply hidden in the noise of transients in previous investigations. Obviously, correct conclusions on reaction mechanisms are not possible when the basic relaxation effects are not assigned properly.

Some experimental difficulty results from the requirement to correct pyranine transients for contributions from absorbance changes of bacteriorhodopsin itself. This correction must be based on data obtained at the same $\mathrm{pH}$ value, which is not trivial in the absence of buffers. Moreover, bacteriorhodopsin transients are modified in the presence of buffers, because the reactions are coupled with each other. Thus, exact correction requires transients measured under identical experimental conditions.

The relatively slow appearance of protons in the bulk is partly due to the coupling between the intramolecular pumping reaction and the intermolecular release reaction(s). Rate constants derived without explicit consideration of this coupling must lead to erroneous conclusions. Furthermore, time constants observed for a process involving intermolecular reaction steps cannot be compared directly with time constants for an intramolecular process, as in previous publications on proton release by bacteriorhodopsin. The danger of false conclusions from an analysis of kinetic data without correct assignment of the coupled reaction mechanism is demonstrated by the data shown in Figure 4. If the analysis of data would be limited to a bacteriorhodopsin concentration in the range of $30 \mu \mathrm{M}$ and would be based on a simple one-step mechanism, the dependence on the pyranine concentration would suggest a very low rate of proton transfer, because the time constant of the relaxation process with the dominant amplitude is virtually independent of the pyranine concentration under these conditions. Heberle ${ }^{28}$ concluded that proton transfer to pyranine is independent of the pyranine concentration. Apparently the bacteriorhodopsin concentration used in his investigation was limited to this special domain.

In the present publication the term "pumping" refers to the first step of the reaction mechanisms (1) or (2) and does not imply any direct relation to one of the individual steps of the established photocycle. Thus, pumping is used in a simple operative manner to describe the reaction chain initiated by the light pulse and ending with the appearance of protons at the release site. An influence of the proton-transfer reaction on the "pumping" time constant must be expected according to the general rules of kinetics. Experimental evidence for this effect is given by the data shown in Figure 2. However, the exact relation of the present pumping reaction to the steps of the established photocycle remains to be demonstrated. In previous investigations any influence of buffer concentration on one of the time constants of the standard photocycle has not been detected yet.

pK Dependence of Proton Transfer to Buffers and Separate Internal Reaction Step. The experimental data obtained for the proton transfer efficiency of various buffers demonstrate the expected general dependence on the $\mathrm{pK}$ difference, corresponding to that described previously for other cases. ${ }^{25}$ The $\mathrm{pK}$ value indicated by this dependence for the proton donor group (7.6) is clearly higher than the value reported previously ${ }^{13}$ for the release group (5.8). A further analysis with a more extensive set of acceptors would be useful to increase the accuracy, to check a wider range of $\Delta \mathrm{pK}$ values and potential effects of other acceptor parameters. Probably the transfer rate is not only dependent on the $\mathrm{pK}$ difference but also on other parameters of the acceptor, in particular size and charge. Effects of this type may have some influence on the $\Delta \mathrm{pK}$ dependence and, thus, it is hardly possible to estimate the accuracy of the $\mathrm{pK}$ value derived from the rate constants obtained in the present investigation. However, in a nonequilibrium system like photoactivated bacteriorhodopsin a difference between a $\mathrm{pK}$ value obtained from rate constants and that obtained from other data is not at all evidence for an error. It is very likely that the difference simply reflects different reaction states of the nonequilibrium system. The $\mathrm{pK}$ value determined in the present investigation clearly is a "kinetic" $\mathrm{pK}$. The nature of this $\mathrm{pK}$ should be analyzed in further investigations. More information may be also obtained by an analysis of the $\mathrm{pH}$ dependence of the proton release kinetics.

Special buffer effects on electric signals of light-excited bacteriorhodopsin were reported by Liu et al. ${ }^{29}$ and TothBoconadi et al. ${ }^{30}$ These effects have been classified according to the charge transition upon protonation. Because the electric signals were measured under different conditions (low salt), a direct comparison to the present results is not possible. In view of the photoelectric results, it is remarkable how closely the proton-transfer rate constants $k_{1}^{+}$obtained for buffers of different charge state follow the expected $\mathrm{pK}$ dependence (Figure 6). Apparently the influence of the acceptor charge is reduced at the much higher salt concentration used in the present investigation.

The analysis based on the reaction mechanisms (1) and (2) reveals an unexpectedly low ratio of the rate constants $k_{0}^{+} / k_{0}^{-}$ for the first reaction step. Probably the low value of this "equilibrium" constant obtained in the presence of many 


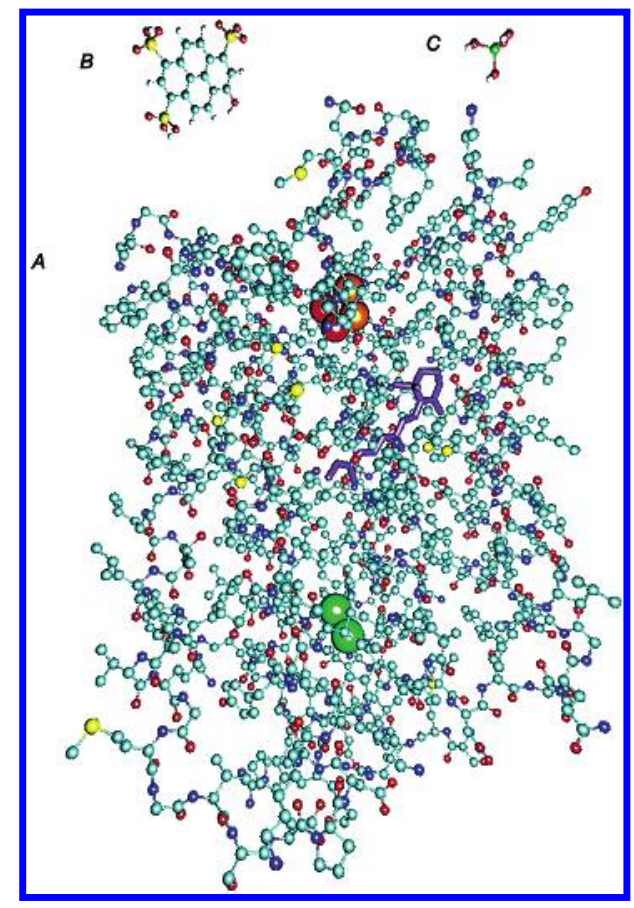

Figure 7. Structures of bacteriorhodopsin (A), pyranine (B), and borate (C) on the same scale. The oxygens of the carboxyl groups of Glu204 (red) + Glu 194 (orange), representing the release group (cf. Discussion), and of Asp96 (green) are highlighted using van der Waals dimensions. The retinal group is presented in "licorice" form (purple). The crystal structure 1c3w.pdb ${ }^{37}$ from the Protein Data Bank ${ }^{39}$ was used for preparation of this figure by VMD..$^{40}$

different proton acceptors is due to a simplified mechanism. An explanation may be based on the large differences in the structure of proton acceptors. An extreme case is pyranine with a bulky aromatic structure and many negative charges, both of which may contribute to exclusion from the cavity of the release domain. Apparently molecules such as pyranine can accept protons only from the periphery of the protein. This implies the existence of a separate proton transfer step from the release cavity to the periphery. It is also possible that access to the release site is enabled by a local change of the protein structure. The identity of the release site is not exactly known. The residues Glu204, ${ }^{31}$ Glu194+Glu204, ${ }^{32}$ a water molecule, ${ }^{33,34}$ and a hydrogen-bonded continuum in this region ${ }^{35,36}$ have been suggested. Crystal structures of bacteriorhodopsin $\sin ^{32,37,38}$ indicate that the residues in the region of the release site are not directly accessible for bulky proton acceptors such as pyranine (Figure 7). Luecke et al. ${ }^{37}$ wrote that the "proton release complex is well insulated from the aqueous medium" and, thus, seemed to anticipate the existence of a separate reaction step from their analysis of the protein structure. However, the present results also indicate that small acceptors such as borate have direct access to this complex.

Another factor contributing to the special release kinetics may be a high degree of hydrogen bonding within the release group. ${ }^{36,41}$ Internal hydrogen bridges are known to decrease proton-transfer rates. ${ }^{42}$ The release group may exist in different states of hydrogen bonding. Transfer of protons may require a separate reaction step for some acceptors. The kinetic models used in the present investigation were not extended yet in general, because additional information should be collected for specification.

Effect of Bacteriorhodopsin Concentration and Surface Mobility of Protons. The unexpectedly strong dependence of the time constant $\tau_{2}$ on the concentration of bacteriorhodopsin observed in the absence of buffers suggests catalysis of proton transfer by some group on the surface of bacteriorhodopsin disks. When this particular pathway of proton transfer is included in the reaction scheme, the concentration dependence of both $\tau_{1}$ and $\tau_{2}$ can be described at a high accuracy (Figure 4 ). The existence of groups serving as proton acceptors and donors on the surface of bacteriorhodopsin must be expected, because there are many different glutamic and aspartic acid residues and also some arginine and lysine residues. Due to electrostatic interactions it is likely that some of the $\mathrm{pK}$ values are shifted into the range where both acceptor and donor function at neutral $\mathrm{pH}$ is possible. In addition to large numbers of bacteriorhodopsin molecules the purple membrane contains many lipid molecules; the headgroups of these lipids may also serve as proton acceptors and donors.

Transfer of protons by intermolecular exchange between bacteriorhodopsin disks is not required if there is a sufficiently high mobility of protons along the surface, because a general high mobility would serve to equilibrate protonation at all available sites by intramolecular transfer. The general problem of proton mobility along membrane surfaces has been discussed extensively (e.g., refs 43, 44). For the special case of bacteriorhodopsin disks, evidence for a high mobility of protons along the surface has been presented by several independent investigations. However, the experimental results given in these investigations for the same type of reaction are very different: Heberle et al. ${ }^{9}$ report a time constant of $228 \pm 39 \mu \mathrm{s}$ for the appearance of protons at indicators fixed on the intracellular side, whereas the time constants of Alexiev et al. ${ }^{11}$ for the corresponding process are 77,76 , and $74 \pm 5 \mu$ s at positions 101, 105, and 160, respectively. Different transfer time constants have been published for the extracellular side as well: proton arrival was reported with time constants $63 \pm 8 \mu \mathrm{s}^{8}$ and $76 \pm$ $18 \mu \mathrm{s}^{9}$ by an indicator fixed at position 129 of the sequence, with a time constant $71 \pm 4 \mu \mathrm{s}^{11}$ at position 72 and with a time constant $134 \mu \mathrm{s}^{13}$ at positions 129 and 130 . These results indicate a relatively broad distribution of exchange time constants, depending on the position of residues and/or on details of experimental conditions. Thus, a prediction of proton transfer time constants to given residues is hardly possible. Under these conditions a final interpretation of the dependence on the bacteriorhodopsin concentration cannot be presented: neither transfer by a residual buffer contamination nor by contact between disks can be excluded.

A special problem associated with the kinetics of proton transfer from bacteriorhodopsin may result from its disk-like structure involving many protein molecules in close contact. A high local proton concentration is expected transiently at the extracellular surface after pulsed photoexcitation. Under these conditions the distribution of protons in space is more time consuming than in the case of protein monomers without aggregation. The problem is analogous to that encountered in ligand binding to linear polymers, where the diffusion controlled rate constants of binding are dependent on the chain length. ${ }^{45,46}$ A simple estimation based on Einstein's equation suggests that the effect is not detectable in the case of bacteriorhodopsin under usual experimental conditions ( $10 \mu \mathrm{M}$ bacteriorhodopsin, $40 \mu \mathrm{M}$ pyranine, $\mathrm{pH} 8$ ). An experimental test with bacteriorhodopsin disks of different diameters in the range from 0.25 to $1 \mu \mathrm{m}$ did not show any difference in the observed time constants. This is partly due to the fact that pumping of protons is relatively slow ( $\tau \approx 50 \mu \mathrm{s}$ ). More evidence for the conclusion that the special distribution effect resulting from the disk-like structure does not affect the experimental transients comes from the observa- 
tion that the time constants are not dependent on the extent of perturbation in the range from approximately 1 to $10 \%$ photoexcitation of bacteriorhodopsin (within the limits of accuracy $\pm 20 \%$, under standard conditions of the present investigation).

Delay or Acceleration of Proton Transfer by Buffer Residues on the Surface? The present investigation provides new information on proton release in bacteriorhodopsin but also raises new questions. One of the goals of the analysis was an analysis of some potential "barrier" in proton release. The results demonstrate that there is no general barrier against diffusion, but the pathway of proton release is more complex than anticipated. If there is no barrier against diffusion of protons into the bulk, how much of the proton flux generated by pumping may be dissipated into the bulk after pumping? The results of the present investigation clearly illustrate the importance of buffer components for proton dynamics. Thus, the potential problem of dissipation depends on the environmental conditions. It is very likely that the natural environment of halobacteria does not contain high concentrations of buffer species that may contribute to dissipation. However, a high local concentration of residues, which may serve as acceptors and donors, is available on the surface of bacteriorhodopsin. These residues promote a relatively high mobility of protons along the membrane surface.

According to the model presented by Nachliel and Gutman, ${ }^{15}$ the buffering capacity on the surface of bacteriorhodopsin delays proton transfer to the bulk. Experimental data obtained in the present investigation provide evidence against this model. The rate constants of proton transfer from bacteriorhodopsin to acceptors such as borate or ammonia approach the limit of diffusion controlled reactions. In general the function of residues on the surface is expected to depend entirely on their mobility: if these residues are sufficiently mobile to allow mutual approach within distances of few water molecules, protons may be transferred without high activation barriers; however, if the residues are fixed, this type of transfer is not possible. The experimental data indicate that there is a broad distribution of transfer rates, which suggests a correspondingly broad distribution of mobilities. It is very likely that this type of motion is the basis for relatively high transfer rates of protons in the plane of bacteriorhodopsin disks.

In summary, proton transfer from bacteriorhodopsin to acceptors in the bulk depends on the nature of the acceptor. Transfer of protons to simple acceptors such as borate proceeds without any detectable barrier, whereas bulky and charged ligands such as pyranine apparently accept protons only from residues at the periphery of bacteriorhodopsin. Thus, there is evidence for some barrier inhibiting transfer of protons to acceptors such as pyranine, but protons are transferred to acceptors such as borate without any significant barrier.

Acknowledgment. Halobacterium salinarum S9 was kindly provided by Dr. J. Tittor. The technical assistance of $\mathrm{J}$. Wawrzinek is gratefully acknowledged. The author is also indebted to Drs. C. Bernasconi, M. Eigen, and G. Maass for discussions on the kinetics of proton transfer.

\section{References and Notes} $52,587$.

(1) Stoeckenius, W.; Bogomolni, R. A. Annu. Rev. Biochem. 1982
(2) Ottolenghi, M.; Sheves, M., Eds. Isr. J. Chem. 1995, 35, 193.

(3) Haupts, U., J.; Tittor, J.; Oesterhelt, D. Annu. Rev. Biophys. Biomol. Struct. 1999, 28, 367.

(4) Lanyi, J. K., Ed. Biochim. Biophys. Acta 2000, 1460, 1.

(5) Lozier, R. H.; Niederberger, W.; Bogomolni, R. A.; Hwang, S.; Stoeckenius, W. Biochim. Biophvs. Acta 1976, 440, 545.

(6) Drachev, L. A.; Kaulen, A. D.; Skulachev, V. P. FEBS Lett. 1984, 178,331 .

(7) Grzesiek, S.; Dencher, N. A. FEBS Lett. 1986, 208, 337.

(8) Heberle, J.; Dencher, N. A. Proc. Natl. Acad. Sci. U.S.A. 1992, 89, 5996.

(9) Heberle, J.; Riesle, J.; Thiedemann, G.; Oesterhelt, D.; Dencher, N. A. Nature 1994, 370, 379 .

(10) Scherrer, P.; Alexiev, U.; Marti, T.; Khorana, H. G.; Heyn, M. P. Biochemistry 1994, 33, 13684.

(11) Alexiev, U.; Mollaaghababa, R.; Scherrer, P.; Khorana, H. G.; Heyn, M. P. Proc. Natl. Acad. Sci. U.S.A. 1995, 92, 372.

(12) Varo, G.; Lanyi, J. K. Biochemistry 1990, 29, 6858.

(13) Zimanyi, L.; Varo, G.; Chang, M.; Ni, B.; Needleman, R.; Lanyi, J. K. Biochemistry 1992, 31, 8535.

(14) Cao, Y.; Brown, L. S.; Sasaki, J.; Maeda, A.; Needleman, R.; Lanyi, J. K. Biophys. J. 1995, 68, 1518.

(15) Mitchell, P. Nature 1961, 191, 144.

(16) Nachliel, E.; Gutman, M. FEBS Lett. 1996, 393, 221.

(17) Oesterhelt, D.; Stoeckenius, W. Methods Enzymol. 1974, XXXI, 667.

(18) Rigler, R.; Rabl, C.; Jovin, T. M. Rev. Sci. Instrum. 1974, 45, 580.

(19) Diekmann, S.; Hillen, W.; Morgeneyer, B.; Wells, R. D.; Porschke,

D. Biophys. Chem. 1982, 15, 263.

(20) Porschke, D.; Jung, M. J. Biomol. Struct. Dvn. 1985, 2, 1173.

(21) Nelder, J.; Mead, R. Comput. J. 1965, 7, 308.

(22) Ilgenfritz, G. Mol. Biol. Biochem. Biophvs. 1977, 24, 1.

(23) Desoye, G.; Porschke, D. Biophys. Chem. 1993, 46, 283.

(24) Wolfbeis, O. S.; Fürlinger, E.; Kroneis, H.; Marsoner, H. Fresenius Z. Analv. Chem. 1983, 314, 119.

(25) Eigen, M. Angew. Chem., Int. Ed. 1964, 3, 1.

(26) Maass, G. Dissertation. Universität Göttingen, 1962.

(27) Steinhoff, H. J.; Pfeiffer, M.; Rink, T.; Burlon, O.; Kurz, M.; Riesle, J.; Heuberger, E.; Gerwert, K.; Oesterhelt, D. Biophys. J. 1999, 76, 2702.

(28) Heberle, J. Dissertation, Freie Universität Berlin, 1991.

(29) Liu, S. Y.; Kono, M.; Ebrey, T. G. Biophvs. J. 1991, 60, 204. 3170 .

(30) Toth-Boconadi, R.; Der, A.; Keszthelyi, L. Biophys. J. 2000, 78,

(31) Brown, L. S.; Sasaki, J.; Kandori, H.; Maeda, A.; Needleman, R.; Lanyi, J. K. J. Biol. Chem. 1995, 270, 27122.

(32) Essen, L. O.; Siegert, R.; Lehmann, W. D.; Oesterhelt, D. Proc. Natl. Acad. Sci. U.S.A. 1998, 95, 11673.

(33) Luecke, H.; Richter, H. T.; Lanyi, J. K. 1998, 280, 1934.

(34) Braiman, M. S.; Mogi, T.; Marti, T.; Stern, L. J.; Khorana, H. G.; Rothschild, K. J. Biochemistry 1988, 27, 8516.

(35) Le Coutre, J.; Tittor, J.; Oesterhelt, D.; Gerwert, K. Proc. Natl. Acad. Sci. U.S.A. 1995, 92, 4962.

(36) Rammelsberg, R.; Huhn, G.; Lübben, M.; Gerwert, K. Biochemistry 1998, $37,5001$.

(37) Luecke, H.; Schobert, B.; Richter, H. T.; Cartaillier, J. P.; Lanyi, J. K. J. Mol. Biol. 1999, 291, 899.

(38) Sass, H. J.; Bueldt, G.; Gessenich, R.; Hehn, D.; Neff, D.; Schlesinger, R.; Berendzen, J.; Ormos, P. Nature 2000, 406, 649.

(39) Berman, H. M.; Westbrook, J.; Feng, Z.; Gilliland, G.; Bhat, T. N.; Weissig, H.; Shindyalov, I. N.; Bourne, P. E. Nucleic Acids Res. 2000, $28,235$.

(40) Humphrey, W.; Dalke, A.; Schulten, K. J. Mol. Graph. 1996, 14, 33.

(41) Spassov, V. Z.; Luecke, H.; Gerwert, K.; Bashford, D. J. Mol. Biol. 2001, 312, 203.

(42) Schuster, P.; Wolschann, P.; Tortschanoff, K. Mol. Biol. Biochem. Biophvs. 1977, 24, 107.

(43) Teissie, J.; Gabriel, B.; Prats, M. TIBS 1993, 18, 243.

(44) Gutman, M.; Nachliel, E. Biochim. Biophvs. Acta 1995, 1231, 123.

(45) Porschke, D. Biophvs. Chem. 1979, 10, 1.

(46) Lohman, T. M. CRC Crit. Rev. Biochem. 1985, 19, 191. 